\title{
Determination of the detective quantum efficiency of a prototype, megavoltage indirect detection, active matrix flat-panel imager
}

\author{
Youcef El-Mohri, ${ }^{\text {a) }}$ Kyung-Wook Jee, Larry E. Antonuk, Manat Maolinbay, \\ and Qihua Zhao \\ Department of Radiation Oncology, University of Michigan Medical Center, Ann Arbor, Michigan 48109
}

(Received 19 April 2001; accepted for publication 28 August 2001)

\begin{abstract}
After years of aggressive development, active matrix flat-panel imagers (AMFPIs) have recently become commercially available for radiotherapy imaging. In this paper we report on a comprehensive evaluation of the signal and noise performance of a large-area prototype AMFPI specifically developed for this application. The imager is based on an array of $512 \times 512$ pixels incorporating amorphous silicon photodiodes and thin-film transistors offering a $26 \times 26 \mathrm{~cm}^{2}$ active area at a pixel pitch of $508 \mu \mathrm{m}$. This indirect detection array was coupled to various x-ray converters consisting of a commercial phosphor screen (Lanex Fast B, Lanex Regular, or Lanex Fine) and a $1 \mathrm{~mm}$ thick copper plate. Performance of the imager in terms of measured sensitivity, modulation transfer function (MTF), noise power spectra (NPS), and detective quantum efficiency (DQE) is reported at beam energies of 6 and $15 \mathrm{MV}$ and at doses of 1 and 2 monitor units (MU). In addition, calculations of system performance (NPS, DQE) based on cascaded-system formalism were reported and compared to empirical results. In these calculations, the Swank factor and spatial energy distributions of secondary electrons within the converter were modeled by means of EGS4 Monte Carlo simulations. Measured MTFs of the system show a weak dependence on screen type (i.e., thickness), which is partially due to the spreading of secondary radiation. Measured DQE was found to be independent of dose for the Fast B screen, implying that the imager is input-quantum-limited at $1 \mathrm{MU}$, even at an extended source-to-detector distance of $200 \mathrm{~cm}$. The maximum DQE obtained is around 1\%-a limit imposed by the low detection efficiency of the converter. For thinner phosphor screens, the DQE is lower due to their lower detection efficiencies. Finally, for the Fast B screen, good agreement between calculated and measured DQE was observed. (c) 2001 American Association of
\end{abstract} Physicists in Medicine. [DOI: 10.1118/1.1413516]

Key words: active matrix flat-panel imager, electronic portal imager, detective quantum efficiency, amorphous silicon

\section{INTRODUCTION}

Following the initial conception of active matrix, flat-panel imagers (AMFPIs) in the late 1980s, ${ }^{1}$ a very large amount of effort has been expended toward the research and development of this x-ray technology for applications in radiology, radiotherapy, and nondestructive testing. These efforts have been directed toward two categories of devices, "direct" 2 and "indirect" 3 detection AMFPIs, that differ in terms of the method of x-ray detection. As a result of these large investments, the last few years have witnessed the widespread commercial introduction of clinically practical direct and indirect detection AMFPIs for a variety of diagnostic imaging applications. More recently, the first commerical AMFPIs specifically designed for imaging radiotherapy treatment beams have been introduced. ${ }^{4}$ These initial radiotherapy products and prototypes rely exclusively on indirect detection of the incident radiation (typically employing a thin metal sheet + a phosphor screen $x$-ray converter), although therapy products based on direct detection are likely to follow.

Based on results from research prototypes, potential improvements in image quality offered by indirect detection AMFPIs, particularly over conventional radiotherapy film systems (the current gold standard), have previously been documented ${ }^{3,5-7}$ as have been the basic signal, noise, and radiation damage resistance properties of these devices. ${ }^{8-17}$ For such systems, there have also been a limited number of detailed, quantitative investigations of higher-order performance variables. The presampling modulation transfer function (MTF) for an AMFPI incorporating a $128 \times 128$ pixel, $750 \mu \mathrm{m}$ pitch, array has been reported by Earnhart and Chaney. ${ }^{18}$ In each pixel of this $9.6 \mathrm{~cm} \times 9.6 \mathrm{~cm}$ array design, a switch consisting of a hydrogenated amorphous-silicon thin-film transistor ( $a-\mathrm{Si}: \mathrm{H}$ TFT) is coupled to a Schottky diode. (Most indirect detection AMFPIs incorporate $a-\mathrm{Si}: \mathrm{H}$ nip photodiodes, rather than a Schottky diode, for reasons of superior dark current behavior.) An initial signal analysis of an AMFPI based on the same array design was reported by Drake et al. ${ }^{19} \mathrm{~A}$ more comprehensive empirical analysis of the linearity, MTF, noise power spectrum (NPS), and detective quantum efficiency (DQE) of another AMFPI, again based on the same array design, has been reported by Munro and Bouius. ${ }^{20}$ This important analysis demonstrated that, unlike TV camera-based electronic portal imaging devices where noise from the camera limits performance, the indirect detection AMFPI studied is $\mathrm{x}$-ray quantum noise limited so 
that its signal-to-noise characteristics are determined by the quantum efficiency of the metal plate/phosphor screen and not by the photodiodes or the readout electronics.

In the present paper, an empirical and theoretical investigation of the signal, NPS, and DQE properties of an AMFPI based on an array, whose design is representative of the current state of the technology, is reported. Empirical results derived from three phosphor screens at two energies are compared with the predictions from a model based on cascaded systems theory. ${ }^{21}$ This study contributes to the limited amount of such observer-independent performance data thus far published for this new portal imaging technology. Beyond the intrinsic value of such characterization information, such results should prove useful for a future comparison with performance data from commercial systems. The present study also provides an examination of the degree to which a cascaded systems model and its associated parameters are able to reproduce the performance of AMFPI systems under a range of therapy imaging conditions. Such comparisons assist in the long-term objective of determining the degree to which this model is able to accurately model AMFPI systems. The AMFPI configurations and imaging conditions reported in the present study partially overlap with those used in an earlier observer-dependent study involving the same array design. ${ }^{6}$

\section{METHODS AND MATERIALS}

\section{A. System description}

The indirect detection AMFPI employed in the present study consists of four major components: a pixelated array that incorporates amorphous silicon TFTs and photodiodes; an x-ray converter placed directly on the surface of the array; a system of acquisition electronics that controls the operation of the array and processes analog pixel data, ${ }^{22}$ and a host computer that controls the acquisition electronics and handles the digital pixel data. Details of the general structure and operation of such imagers can be found in Refs. 5 and 23. In this study, the x-ray converter consisted of a phosphor screen coupled to an overlying metal plate ( $\sim 1 \mathrm{~mm}$ copper). A total of three commercially available screens (each containing $\left.\mathrm{Gd}_{2} \mathrm{O}_{2} \mathrm{~S}: \mathrm{Tb}\right)$ were used: Lanex Fast $\mathrm{B}(\sim 133$ $\left.\mathrm{mg} / \mathrm{cm}^{2}\right)$, Lanex Regular $\left(\sim 70 \mathrm{mg} / \mathrm{cm}^{2}\right)$, and Lanex Fine $\left(\sim 34 \mathrm{mg} / \mathrm{cm}^{2}\right)$ (Eastman Kodak). In megavoltage imaging, the presence of the metal plate serves to reduce scatter and to convert incident $\mathrm{x}$ rays into high-energy electrons, functioning as a form of buildup layer.

The array has a $512 \times 512$ pixel format corresponding to a $26 \times 26 \mathrm{~cm}^{2}$ active area with a pixel pitch of $508 \mu \mathrm{m}$. Each pixel consists of a nip photodiode coupled to a thin-film transistor (TFT). The fraction of the pixel area occupied by the photodiode (fill factor) is 0.83 . While the photodiode constitutes the charge-collecting element in the pixel, the TFT acts as a switch enabling charge readout by peripheral electronics. The array pixels are arranged in a twodimensional matrix of rows and columns. The gate contacts of all pixel TFTs along a given row are connected to a common conductive trace, called a "gate line." The drain con-

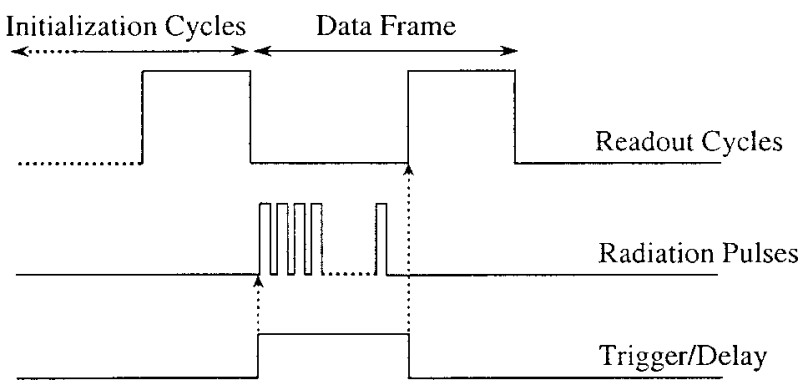

FIG. 1. Timing diagram illustrating the synchronization of array readout with radiation beam delivery for radiographic operation.

tacts of all pixel TFTs along a given column are connected to a common conductive trace, referred to as a "data line." During imaging, the radiation signal created by incident $\mathrm{x}$ rays is integrated in the capacitance of the photodiodes (to which a reverse bias voltage of $-5 \mathrm{~V}$ is applied) by keeping the TFTs nonconducting through the application of a negative voltage $(-8 \mathrm{~V})$ to all gate lines. Readout of the imaging signal is performed one row of pixels at a time by making the TFTs conducting through the application of a positive voltage $(+10 \mathrm{~V})$ to the corresponding gate line. The pixel signal is integrated in peripheral charge sensitive preamplifiers. Analog signals from the preamplifiers are multiplexed and digitized to an effective resolution of 15 bits. The readout of all, or some fraction, of the rows is termed a "readout cycle" and the corresponding information, if saved, is termed a "data frame." For all measurements, a data acquisition sequence consists of acquiring a number of consecutive readout cycles. The information for the first (typically 50) readout cycles is not saved. Following these "initialization cycles," data frames are acquired for the measurements. The frame time, defined as the period between consecutive readout cycles, is varied in order to accommodate different irradiation times.

\section{B. General experimental conditions}

All measurements were performed with a Clinac-1800 linear accelerator (Varian Associates) using 6 and $15 \mathrm{MV}$ photon beams calibrated such that $1 \mathrm{MU}$ (Monitor Unit) delivers $1 \mathrm{cGy}$ of dose at $100 \mathrm{~cm}$ from the source at a depth of maximum dose $\left(d_{\max }\right)$ in water for a $10 \times 10 \mathrm{~cm}^{2}$ field. The accelerator was operated at $400 \mathrm{MU} / \mathrm{min}$. The imager was operated in radiographic mode for most of the studies except for sensitivity measurements, where fluoroscopic acquisition was used. In radiographic operation, array readout was synchronized with the radiation source in order to ensure that each row of pixels was exposed to the same amount of radiation per data frame. As seen in Fig. 1, such synchronization was achieved by means of a trigger/delay pulse generated by the acquisition electronics. ${ }^{6}$ The leading edge of this pulse provides a trigger for the start of radiation delivery and is issued following the last initialization cycle. The trailing edge of the pulse follows the end of the irradiation and provides a trigger for the start of array readout (i.e., capture of the data frame). The width of the pulse is adjusted so as to 
accommodate the duration of the entire irradiation. The initialization cycles performed prior to the irradiation serve to remove trapped charge that accumulates in the photodiodes when the array is not read out. In fluoroscopic operation, there was no synchronization between array readout and radiation delivery. In addition, in this case only a single row of pixels was read out per cycle so as to maximize the range of achievable frame times. For all measurements, the surface of the array was positioned vertically and the accelerator gantry was rotated to an angle of $90^{\circ}$. This was done to reduce the effect of scatter from the floor-since a horizontal detector configuration would result in a large backscatter radiation component, especially at the extended source-to-detectordistance, SDD, used.

\section{Sensitivity}

The determination of sensitivity (signal response per unit incident radiation) is of inherent interest as it represents an important metric of system performance. Moreover, in the present study, sensitivity information for three imager configurations, corresponding to the various phosphor screens, was used to determine the values of a gain factor needed in the cascaded systems model that was otherwise unknown (see Sec. II F). Such determinations were achieved by comparing model predictions to measurements of sensitivity. Sensitivity data were acquired at 6 and $15 \mathrm{MV}$ with a SDD of $\sim 200 \mathrm{~cm}$ and a collimator setting of $15 \times 15 \mathrm{~cm}^{2}$. The use of the extended distance was to avoid saturation of the preamplifiers by the large light output of the Fast-B screen. The imager was operated in fluoroscopic mode for frame times ranging from $\sim 0.045$ to $\sim 1.63 \mathrm{~s}$, varied by means of software-controlled delays. For each data acquisition sequence, a total of 100 data frames were obtained and averaged to yield pixel signal. These frames were preceded by a number of initialization cycles, which were performed while radiation was continuously being delivered. This initialization process was used to achieve equilibrium between charge trapping and charge release, thereby eliminating the effect of charge trapping. ${ }^{23}$ The measurement of sensitivity consisted of acquiring signal data for a collection of pixels as a function of irradiation time (MU). In the measurements, signal size was kept below $15 \%$ of pixel saturation, thereby ensuring highly linear signal response. ${ }^{6}$ Following the subtraction of dark signal data obtained at the same frame time, an average response of the pixels was obtained, the slope of which yielded the sensitivity in units of electrons per MU at the SDD of the detector.

\section{Modulation transfer function (MTF)}

MTFs of the imaging system were measured for the three converters using a pair of steel blocks, each of dimension $40 \times 20 \times 5 \mathrm{~cm}^{3}$. Opposing $40 \times 20 \mathrm{~cm}^{2}$ machined surfaces on the blocks were separated by shims to form a narrow, $\sim 100$ $\mu \mathrm{m}$ wide, slit. The blocks were placed in front of the imager forming a slit of dimensions $200 \times 0.1 \mathrm{~mm}^{2}$ and acting as a $40 \mathrm{~cm}$ long radiation collimator. Both the imager and the blocks were placed on a micropositioning (translation) table allowing alignment of the slit with the center of the radiation beam (for maximum imager signal). The array and overlying converter, positioned at a SDD of $\sim 130 \mathrm{~cm}$, were in direct contact with the exit surface of the steel blocks. Since the narrowness of the slit greatly limited the fluence transmitted through it, long irradiations were required to produce slit images. These irradiations ranged from 10 to $70 \mathrm{MU}$, depending on the converter used. The array was operated in radiographic mode and frame time was varied from 2.4 to $11.5 \mathrm{~s}$ in order to accommodate the delivery of radiation prior to array readout. In all MTF measurements, the slit was oriented at a small angle $\left(\sim 1^{\circ}\right)$ with respect to the data lines on the array, so as to allow oversampling of the line spread function (LSF). This is referred to as the angled-slit technique. ${ }^{24}$ In this manner, sampling at intervals of $\sim 10 \mu \mathrm{m}$ was achieved.

For each converter, a total of five data frames, each corresponding to a region of 256 data lines by 512 gate lines, were acquired and averaged to yield an image of the slit. For all slit images, gain and offset corrections ${ }^{3}$ were applied in order to correct for stationary variations in pixel response, for channel-to-channel variations among the preamplifier electronics, and for structure noise inherent to the phosphor screen. These gain and offset corrections were derived from the average of ten dark and ten flood-field frames obtained under conditions similar to those of the slit images, but in the absence of the slit. In order to maintain a pixel signal level similar to that of the slit images, so as to remain within the linear region of the pixel response, ${ }^{6}$ shorter irradiation times were used (1 MU for the Fast B and Regular screens, and 3 MU for the Fine screen). Additional slabs of solid water were used in front of the collimator to further reduce the signal size, as necessary. For each converter and beam energy, the final, corrected slit images were used to determine the LSF. After an application of baseline subtraction and normalization corrections, the Fourier transforms of each LSF yielded a MTF.

\section{E. Noise power spectra (NPS)}

NPS measurements of the imaging system were performed for the Fast B screen at 6 and $15 \mathrm{MV}$ (at 1 and 2 $\mathrm{MU}$ ) and for the Regular and Fine screens at $6 \mathrm{MV}$ (at 2 MU). Although the thicker screen (Fast B) is most appropriate for megavoltage energies, ${ }^{6}$ exploring thinner screens serves to illustrate the range of validity of the cascaded systems model. In these measurements, the imager was placed at a SDD of $200 \mathrm{~cm}$ with a collimator setting of $15 \times 15 \mathrm{~cm}^{2}$. The imager was operated radiographically with a trigger/ delay pulse of $\sim 0.5 \mathrm{~s}$ to accommodate the delivery of 1 or 2 MU of radiation. The frame time was $\sim 2.5 \mathrm{~s}$. For each converter, energy, and irradiation, a total of 20 data frames were acquired, with $512 \times 422$ pixels (data $\times$ gate) per frame. An analysis was performed to a central region of the array consisting of $256 \times 256$ pixels. For each converter and energy, two types of NPS data were acquired. The first type consisted of regular flood-field data while the second type was obtained with the addition of a thin opaque layer of paper 


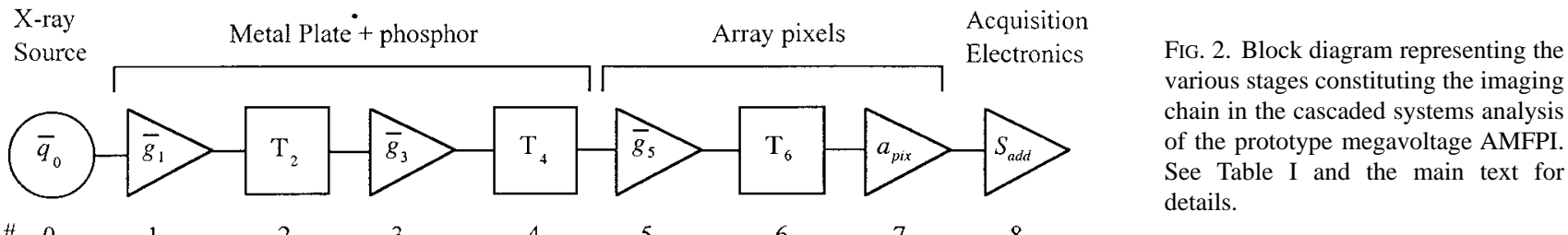

Stage \# 0

1

5

8

between the array surface and the phosphor screen. This configuration was employed to stop optical photons from reaching the array, thus giving a measure of NPS in the absence of optical photons (referred to as dark NPS). Dark NPS includes system noise in the absence of radiation as well as non-screen-related effects caused by the radiation source. Measurement of dark NPS provided an estimate of the total additive noise - an empirically determined component required in the cascaded systems NPS calculations (see the next section). For each measured NPS, corresponding gain and offset corrections were obtained and applied to the data. A $3 \times 3$ median filter was also applied to correct for defective pixels, which represent less than $0.5 \%$ of the total number of pixels. After converting pixel signal values into electrons by means of a measured calibration factor for the preamplifiers ( $1 \mathrm{ADC} \approx 10000 \mathrm{e}^{-}$), NPS were determined using a synthesized slit technique. ${ }^{25-27}$ This technique involved the selection of 160 independent, nonoverlapping "slits" from the measured data frames. Each slit, consisting of data from a $32 \times 256$ pixel block, was oriented along the gate line direction. Each slit was summed along the data line direction to form a 256-point realization. After subtraction of lowfrequency background trends and the application of a Hanning window function, a Fourier transform was applied to each of the 160 realizations. The results were then appropriately normalized to yield an ensemble of 160 power spectra, the average of which yielded the measured NPS. The measured NPS was used to empirically determine the detective quantum efficiency (DQE), as explained in the next section.

\section{F. Cascaded systems model}

Theoretical modeling of $\mathrm{x}$-ray imagers serves to contribute toward an understanding of the properties, limitations and potential performance of such devices. In particular, considerable success has been demonstrated in using a cascaded systems analysis to describe signal and noise performance of both direct $^{2}$ and indirect ${ }^{28,29}$ detection AMFPIs at diagnostic energies as well as of video-based MV systems. ${ }^{30,31}$ In this analysis, the imaging system is divided into a series of stages where each stage represents a physical process possessing its own gain, noise, and spatial spreading properties. Each stage can be characterized by an intrinsic gain $\left(\overline{g_{i}}\right)$, a gain variance $\left(\sigma_{g i}^{2}\right.$, expressed in terms of the Poisson excess, $\epsilon_{g i}$, by the relation $\epsilon_{g i}=\left[\sigma_{g i}^{2} / \overline{g_{i}}\right]-1$, or in terms of the Swank factor), and the MTF $\left[T_{i}(u, \nu)\right]$, where $u$ and $\nu$ are orthogonal spatial frequency coordinates. The signal and noise transfer properties of the imager are determined by the transfer properties of each stage. ${ }^{32}$ Figure 2 shows a simplified diagram illustrating the various stages in the imaging system used in this study while the parameters describing the system are summarized in Table I. Stage 0 represents the incident $\mathrm{x}$ rays characterized by an incident fluence $\overline{q_{0}}$. Stage 1 represents the selection of interacting $\mathrm{x}$ rays in the converter with a quantum efficiency $\overline{g_{1}}$. Almost all interactions are due to Compton scatter, and only the scattered electrons deposit significant energy in the phosphor. Stage 2 represents the process of determining where the Compton electron deposits its energy. This is accomplished by the application of a scatter operator $^{33,34}$ that relocates image quanta by a random vector characterized in terms of the point-spread function having a MTF described by $T_{2}(u, \nu)$. Stage 3 represents the generation of optical photons that will be emitted from the converter with a gain $\overline{g_{3}}$. Stage 4 represents the spread of these optical photons within the converter characterized by a MTF, $T_{4}(u, \nu)$. Stage 5 represents the coupling of optical photons to the photodiode with a gain $\overline{g_{5}}$. Stage 6 represents the

TABLE I. Stages, processes, and associated parameters used in the cascaded systems analysis of the prototype megavoltage AMFPI examined in this paper. Note that this characterization generally applies to any indirect detection megavoltage AMFPI.

\begin{tabular}{cll}
\hline \hline Stage \# & \multicolumn{1}{c}{ Description } & \multicolumn{1}{c}{ Parameters } \\
\hline$i=0$ & X rays incident upon detector & $\overline{q_{0}}:$ Incident fluence \\
$i=1$ & Interaction of x rays in converter & $\overline{g_{1}}:$ Quantum detection efficiency \\
$i=2$ & Spatial spreading of secondary radiation in converter & $T_{2}(u, \nu)$ : Secondary radiation MTF \\
$i=3$ & Generation and emission of optical quanta in converter & $\overline{g_{3}}:$ Quantum gain \\
& & $\epsilon_{g_{3}}:$ Poisson excess \\
$i=4$ & Spatial spreading of optical quanta in converter & $T_{4}(u, \nu):$ Optical screen MTF \\
$i=5$ & Coupling of optical quanta to photodiode & $\overline{g_{5}}:$ Optical coupling efficiency \\
$i=6$ & Integration of optical quanta by photodiode & $T_{6}(u, \nu):$ Photodiode MTF \\
& & $a_{\mathrm{pd}}:$ Photodiode aperture \\
$i=7$ & Sampling of array pixels & $a_{\mathrm{pix}}:$ Pixel pitch \\
$i=8$ & Pixel readout & $S_{\mathrm{add}}(u, \nu)$ : Additive electronic noise \\
\hline \hline
\end{tabular}


integration of optical quanta by a square photodiode aperture (with a side of length $a_{\mathrm{pd}}$ ) characterized by a MTF, $T_{6}(u, \nu)$. Stage 7 represents sampling of the signal from each pixel having a pixel-to-pixel pitch of $a_{\text {pix }} \cdot a_{\text {pix }}$ is larger than $a_{\mathrm{pd}}$ and they are related by $a_{\mathrm{pd}}^{2}=a_{\text {pix }}^{2} \times$ fill factor. Finally, stage 8 represents readout of the imaging signal by the acquisition electronics characterized by an additive noise, $S_{\text {add }}(u, \nu)$.

In comparison with previous analyses conducted on indirect detection imagers at diagnostic energies, ${ }^{28,29}$ stage 2 has been added since, at megavoltage energies, the spread created by a single secondary Compton electron from each interacting high-energy $\mathrm{x}$ ray within the converter is not negligible. ${ }^{30}$ This is still a simplification, as it assumes that for each interaction the Compton electron deposits its energy (and generates light) at a single point. In fact, as secondary electrons travel within the phosphor, they deposit energy while simultaneously generating light photons along their path. The simplifying assumption in the model is that interacting, incident quanta are spatially relocated to an average position along the path of the ensuing secondary electrons within the phosphor screen-a process estimated through Monte Carlo simulations (see Sec. II F 2). The following sections gives the cascaded systems expressions for sensitivity, NPS, and DQE corresponding to the model outlined above.

\section{Sensitivity}

The x-ray sensitivity of the imaging pixels, $\Gamma$, involves a linear combination of the system gain factors:

$$
\Gamma=\left(\overline{\frac{q_{0}}{D}}\right) a_{\mathrm{pd}}^{2} \overline{g_{1}} \overline{g_{3}} \overline{g_{5}}, \quad(\mathrm{e} / \mathrm{MU} / \mathrm{pixel}),
$$

where $\overline{q_{0}}$ is the fluence of incident radiation (photons per unit area), $D$ is the irradiation time in units of $\mathrm{MU}$, and $a_{\mathrm{pd}}$ is the size of the photodiode. The fluence was estimated from the tables in Ref. 35, using energy spectra (at 6 and $15 \mathrm{MV}$ ) taken from Ref. 36. The average optical coupling efficiency $\left(\overline{g_{5}}\right)$ was determined from the product of the phosphor $\left(\mathrm{Gd}_{2} \mathrm{O}_{2} \mathrm{~S}: \mathrm{Tb}\right)$ emission spectrum and the absorption spectrum of the array photodiode, ${ }^{23}$ resulting in a value for $\frac{p}{g_{5}}$ of 0.65 for all screens. The detection efficiency of the converter $\left(\overline{g_{1}}\right)$ was obtained from EGS4 Monte Carlo simulations based on the geometry and physics reported in Ref. 37 and spectra taken from Ref. 36. In these simulations, a user code $\left(\right.$ RZ_PHS $^{37}$ ) simulates photon-electron (positron) transport within a plane-cylindrical geometry and scores the amount of energy deposited within the detector volume for each photon history. The modeled geometry consisted of a pencil beam of photons incident perpendicularly on a $40 \mathrm{~cm}$ diameter planar imaging detector. The detector consisted of a $1 \mathrm{~mm} \mathrm{Cu}$ layer overlying a thickness of $\mathrm{Gd}_{2} \mathrm{O}_{2} \mathrm{~S}$ phosphor. Different phosphor thicknesses were considered, 90, 190 and $360 \mu \mathrm{m}$ corresponding to Lanex Fine, Regular, and Fast B screens, respectively. For all cases, the phosphor layer was modeled as $\mathrm{Gd}_{2} \mathrm{O}_{2} \mathrm{~S}$ with a density of $3.67 \mathrm{~g} / \mathrm{cm}^{3}$. This is approximately $50 \%$ of the bulk density of $\mathrm{Gd}_{2} \mathrm{O}_{2} \mathrm{~S}$. This reduction in density accounts for the polymer binder and small air pockets contained within a realistic phosphor layer. In the simulation, absorbed energy distributions (AEDs) in the phosphor were obtained by scoring the amount of energy deposited by each interacting incident photon. The gain $\overline{g_{1}}$ was obtained by dividing the number of photons that deposit energy in the phosphor by the total number of incident photons.

The quantum gain $\left(\overline{g_{3}}\right)$, defined as the mean number of optical quanta exiting the phosphor screen per interacting $\mathrm{x}$ ray, was calculated using the expression

$$
\overline{g_{3}}=\eta \frac{E_{\mathrm{ab}}}{E_{\mathrm{opt}}}
$$

where $\eta$ is the efficiency of the phosphor screen in converting energy deposited by x rays into optical photons. (In this definition, $\eta$ includes both the intrinsic screen conversion efficiency and the probability of optical photons exiting the screen.) $E_{\text {opt }}$ is the mean energy of optical photons emitted by the screen $(2.3 \mathrm{eV})^{38}$ and $E_{\mathrm{ab}}$ is the mean energy absorbed per interacting x ray. $E_{\mathrm{ab}}$ was determined from the AEDs obtained from the EGS4 Monte Carlo simulations described above. ${ }^{37}$ In the expression for $\overline{g_{3}}$, the screen efficiency $(\eta)$ is the only unknown parameter. Due to the difficulty in estimating this quantity, given the absence of measured data or calculations at megavoltage energies in the literature, $\eta$ was deduced by fitting the calculated sensitivities to the corresponding measurements for each screen and energy. For both the Fast B and Regular screens, efficiencies of $\sim 0.14$ were deduced at both 6 and $15 \mathrm{MV}$. For the Fine screen, however, $\eta$ was found to be $\sim 0.07$ (half the value of the thicker screens). The difference in efficiency between the screens is likely due to the presence of a reflector at the entrance surface for Fast B and Regular screens, which improves their overall quantum gain $\left(\overline{g_{3}}\right)$. The same factor-of-2 decrease in light output between the two types of screens (i.e., with and without reflector) has also been observed at diagnostic energies, ${ }^{39}$ supporting our empirically deduced values. In this manner, values for $\eta$, and thus $\overline{g_{3}}$, were determined and subsequently used in the calculations of NPS and DQE. Table II gives a summary of the parameter values used in the calculations for the various phosphor screens and energies.

\section{Noise power spectra (NPS)}

Using the cascaded systems formalism and the aforementioned gain, noise, and spreading definitions for the various stages of the imaging system, the following expression for the NPS was derived: ${ }^{29}$

$$
\begin{aligned}
S(u, \nu)= & a_{\mathrm{pd}}^{4} \overline{q_{0}} \overline{g_{1}} \overline{g_{3}} \overline{g_{5}}\left[1+\overline{g_{5}}\left(\overline{g_{3}}+\epsilon_{g_{3}}\right) T_{4}^{2}(u, \nu)\right] \\
& \times T_{6}^{2}(u, \nu)^{* *} \operatorname{III}(u, \nu)+S_{\text {add }}(u, \nu) \quad\left(\mathrm{mm}^{2}\right) .
\end{aligned}
$$

In this formula, the process of sampling (stage 7) is represented by the convolution of the presampled NPS with the Fourier transform of the sampling grid, $\operatorname{III}(u, \nu)$. Thus $S(u, \nu)$ includes the effects of aliasing on the NPS. The sampling grid may be written as 
TABLE II. Summary of numerical values of parameters used in the cascaded systems calculations in this paper. (See the text for details.)

\begin{tabular}{ccccc}
\hline \hline & Lanex Fine & Lanex Regular & \multicolumn{2}{c}{ Lanex Fast B } \\
\cline { 4 - 5 } Term & $(6 \mathrm{MV})$ & $(6 \mathrm{MV})$ & $(6 \mathrm{MV})$ & $(15 \mathrm{MV})$ \\
\hline$\left(\overline{q_{0}} / D\right)\left(\mathrm{photons} / \mathrm{mm}^{2} / \mathrm{MU}\right)$ & $3.46 \times 10^{6}$ & $3.46 \times 10^{6}$ & $3.46 \times 10^{6}$ & $2.14 \times 10^{6}$ \\
$\overline{g_{1}}$ & 0.010 & 0.013 & 0.018 & 0.020 \\
$\overline{g_{3}}$ & 2680 & 9417 & 14424 & 17854 \\
$\frac{\epsilon_{g_{3}}}{g_{5}}$ & 2154 & 7264 & 11867 & 14056 \\
\hline \hline
\end{tabular}

$$
\mathrm{III}(u, \nu)=\sum_{k, l=-\infty}^{\infty} \delta\left(u-k u_{s}, \nu-l \nu_{s}\right) .
$$

In Eq. (4), $u_{s}$ and $\nu_{s}$ correspond to sampling frequencies, given by

$$
u_{s}, \nu_{s}=\frac{1}{a_{\text {pix }}},
$$

where $a_{\text {pix }}$ is the pixel pitch. In Eq. (3), $S_{\text {add }}(u, \nu)$ (also referred to as the dark NPS) corresponds to the noise power spectrum of the total additive noise and includes the effects of pixel dark noise, amplifier noise, correlated noise, and any non-screen-related noise induced by the radiation source that is not accounted for in the model. $S_{\text {add }}(u, \nu)$, an empirical input to the model, was measured in the manner previously described in Sec. II E. The term $\epsilon_{g_{3}}$ in Eq. (3) represents the Poisson excess in $\overline{g_{3}}$ and was derived from the equation ${ }^{21}$

$$
\epsilon_{g_{3}}=\overline{g_{3}}\left(\frac{1}{I}-1\right)-1 \text {, }
$$

where $I$ is the Swank factor obtained from the absorbed energy distribution in the phosphor. ${ }^{37}$ Since no empirical data or theoretical model of light propagation within the phosphor is available, the variance in the absorbed energy distributions was assumed to dominate the Swank factor while any variations caused by optical light transport within the phosphor were neglected. ${ }^{30}$ In Eq. (3), the gain $\overline{g_{5}}$ is simply the coupling efficiency of the photodiode $\left(\overline{g_{5}}\right)$, corrected for signal loss due to charge trapping in the $a$-Si photodiodes. ${ }^{6}$ (Since the NPS measurements were performed radiographically, they are affected by the loss of signal due to charge trapping. Consequently, this correction to $\overline{g_{5}}$ accounts for the effect of charge trapping in the model.) Since charge trapping can be signal dependent, ${ }^{6}$ this charge loss was empirically determined for each measurement configuration (i.e., each converter-energy-irradiation combination) from a comparison of the pixel signal obtained from the NPS flood-field radiographic data and the signal obtained from fluoroscopic sensitivity measurements. ${ }^{23}$

The terms $T_{4}(u, \nu)$ and $T_{6}(u, \nu)$ in Eq. (3) are spreading stages. $T_{6}(u, \nu)$ is simply given by the Sinc function associated with the aperture of each square photodiode (of dimension $\left.a_{\mathrm{pd}}\right) . T_{4}(u, \nu)$ is defined as the optical screen MTF, rep- resenting one of the components of the imaging system MTF. In this representation, the system $\operatorname{MTF}\left[T_{\text {sys }}(u, \nu)\right]$ is assumed to be given by the product

$$
T_{\text {sys }}(u, \nu)=T_{2}(u, \nu) T_{4}(u, \nu) T_{6}(u, \nu),
$$

where contributions from other sources such as focal spot and scatter are negligible due to the collimating blocks used in the MTF measurements. Since $T_{\text {sys }}(u, \nu)$ can be measured, and as $T_{6}(u, \nu)$ is readily calculated, $T_{4}(u, \nu)$ can be derived from $T_{\text {sys }}(u, \nu)$ if $T_{2}(u, \nu)$ can be determined. In order to estimate $T_{2}(u, \nu)$, EGS4 Monte Carlo simulations based on the DOSXYZ user code ${ }^{40}$ were performed. In these simulations, a photon line source with a width of $4 \mu \mathrm{m}$ was incident perpendicular to a converter (with photon energies generated to represent actual energy spectra obtained from Ref. 36. The choice of a $4 \mu \mathrm{m}$ width decreased simulation time while not affecting the resulting MTF at the frequencies of interest. The converter consisted of a $1 \mathrm{~mm} \mathrm{Cu}$ plate overlying a thickness of $\mathrm{Gd}_{2} \mathrm{O}_{2} \mathrm{~S}$ phosphor. Three different phosphor thicknesses were considered, 90, 190, and $360 \mu \mathrm{m}$ corresponding to Lanex Fine, Regular and Fast B screens, respectively. The spatial distribution of the energy deposited in the phosphor was determined by dividing the phosphor into a matrix of small, $2 \times 2 \mu \mathrm{m}$-wide bins with a bin depth corresponding to the phosphor thickness. In this manner, for each case a two-dimensional profile of dose distribution within the phosphor was obtained. From this profile, a one-dimensional dose distribution corresponding to a line spread function (LSF) was obtained from bins lying along a direction perpendicular to the slit direction and intersecting the center of the slit. From this LSF, $T_{2}(u, \nu)$ was calculated by taking the Fourier transform. Subsequently, $T_{4}(u, \nu)$ was derived by dividing the measured MTF of the system by the product of $T_{6}(u, \nu)$ and $T_{2}(u, \nu)$.

\section{Detective quantum efficiency (DQE)}

To predict imaging system performance, the DQE was determined. The DQE gives a measure of how efficient an imaging system is in transferring the information content of the incident radiation from the input to the output of the system. It may be defined as the ratio of the signal to noise at the output of the system to the signal to noise at the input of the system, all squared. Using Eq. (3), the DQE may also be expressed as the ratio of ideal and actual NPS, ${ }^{29}$ which, after suitable manipulation, yields the following expression: 


$$
\operatorname{DQE}(u, \nu)=\frac{\overline{g_{1}} \overline{g_{3}} \overline{g_{5_{T}}} T_{2}^{2}(u, \nu) T_{4}^{2}(u, \nu) T_{6}^{2}(u, \nu)}{\left(\left[1+\overline{g_{5}}\left(\overline{g_{3}}+\epsilon_{g_{3}}\right) T_{4}^{2}(u, \nu)\right] T_{6}^{2}(u, \nu) * * \frac{\mathrm{III}(u, \nu)}{a_{\mathrm{pd}}^{4} \overline{q_{0}} \overline{g_{1}} \overline{g_{3}} \overline{g_{5_{T}}}}\right)+\frac{S_{\mathrm{add}}(u, \nu)}{a_{\mathrm{pd}}^{4} \overline{q_{0}} \overline{g_{1}} \overline{g_{3}} \overline{g_{5_{T}}}}} .
$$

In Eq. (8), all parameters are either directly known, calculated, or empirically determined, as discussed in the previous two sections. As previously discussed, the product of $T_{2}(u, \nu), T_{4}(u, \nu)$, and $T_{6}(u, \nu)$ is assumed to represent the measured system MTF, $T_{\text {sys }}(u, \nu)$. Calculated DQE values obtained from this expression were compared to measured DQE values, which were obtained using the equation

$$
\operatorname{DQE}(u, \nu)=\frac{A^{2} T_{\text {sys }}^{2}(u, \nu)}{\overline{q_{0}} \operatorname{NPS}(u, \nu)},
$$

where $A$ is the mean signal in units of electrons derived from the NPS flood-field data, $\overline{q_{0}}$ is the incident $\mathrm{x}$-ray fluence (photons per unit area), and NPS represents the measured noise power spectrum.

\section{RESULTS}

\section{A. Sensitivity}

Figure 3 shows the measured pixel signal as a function of incident radiation for the various converters at two beam energies. For each imager configuration, the response is linear and the corresponding solid line represents a linear fit from which sensitivity, in units of electrons per MU per pixel, is obtained. Sensitivity values for the various converters at two beam energies are shown in Table III. Imager sensitivity is observed to increase with increasing phosphor screen thickness mainly due to increases in quantum detection efficiency $\left(\overline{g_{1}}\right)$ and quantum gain $\left(\overline{g_{3}}\right)$ (see Table II).

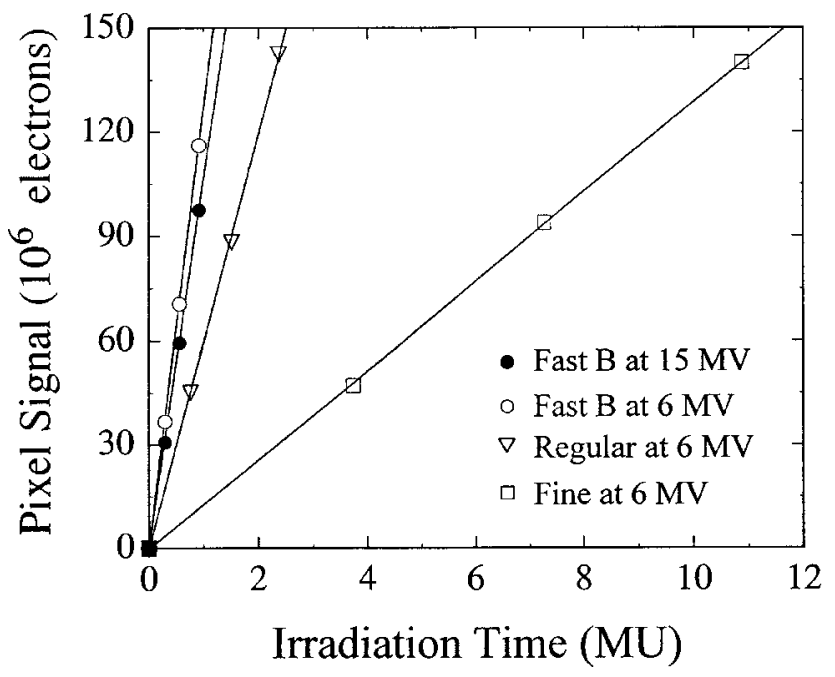

FIG. 3. Pixel response as a function of irradiation time (MU) for various screens and beam energies. At $6 \mathrm{MV}$ beam energy, data is shown for Fast B (open circles), Regular (triangles), and Fine screen (open squares). At 15 MV beam energy, data is shown for a Fast B screen (solid circles).
For the Fast B screen, the $6 \mathrm{MV}$ sensitivity result is higher than that at $15 \mathrm{MV}$, mainly due to the corresponding higher $\mathrm{x}$-ray fluence. This increase in fluence dominates the increase or decrease of the other parameters of Eq. (1). Overall, the magnitude and energy dependence of the present results are generally comparable with those of an earlier study, ${ }^{6}$ after differences in SDD and detector setup are taken into account.

\section{B. Modulation transfer functions}

Figure 4 shows the various modulation transfer functions (MTFs) determined in the present study for each of the three $\mathrm{X}$-ray converters. For each converter, the measured total system MTF $\left(T_{\text {sys }}\right)$, as well as the calculated secondaryradiation MTF $\left(T_{2}\right)$, are shown. The photodiode MTF $\left(T_{6}\right)$ is represented by a Sinc function, which originates from the Fourier transform of the rect function used to represent the photodiode. For each converter, the optical screen MTF was obtained by dividing $T_{\text {sys }}$ by the product of $T_{2}$ and $T_{6}$ and a fit to the result served as input to the NPS calculations. In this case, any contribution due to the slit width $(100 \mu \mathrm{m})$ is ignored since its effect on determining the optical screen MTF is negligible.

A comparison of calculated radiation MTFs $\left(T_{2}\right)$ at $6 \mathrm{MV}$ for the various screens [Figs. 4(a), 4(c), and 4(d)] indicates that thicker screens induce greater spreading due to the extended lateral path of the secondary radiation (electrons) within the phosphor, as expected. Similarly, for a given screen thickness (Fast B), the higher-energy $15 \mathrm{MV}$ beam produces a lower secondary-radiation MTF than the $6 \mathrm{MV}$ beam [Figs. 4(a) and 4(b)] due to the higher energies imparted to electrons within the phosphor+copper material, resulting in increased lateral spreading.

A comparison of the measured total system MTFs for the various screens at $6 \mathrm{MV}$ [Figs. 4(a), 4(c), and 4(d)] indicates that system resolution improves only slightly with thinner phosphor screens (unlike the results obtained at diagnostic energies ${ }^{41}$ ). This is partially due to the additional spreading induced by the secondary electrons (as reflected by $T_{2}$ ) in the megavoltage case. Similarly, comparing total system MTF ( $\left.T_{\text {sys }}\right)$ for the Fast B screen at the two beam energies [Figs. 4(a) and 4(b)] indicates that the use of the lower-energy beam slightly improves the total system MTF through the reduction of lateral spreading of secondary radiation within the converter. The deduced optical screen MTFs $\left(T_{4}\right)$ do not exhibit a strong dependence on screen thickness. This result is unexpected since the optical MTF of the phosphor screen is, in principle, expected to improve with decreasing thickness of the phosphor. The result may be due to the existence of an additional blurring mechanism (not thus far accounted 
TABLE III. Measured pixel sensitivity in units of charge per unit irradiation (MU) at the SDD of the imager (200 $\mathrm{cm}$ ) for various screen thicknesses. Note that the results were obtained in the fluoroscopic mode where the processes of charge trapping and release in the photodiodes are in equilibrium.

\begin{tabular}{lcccc}
\hline \hline Phosphor screen & $\begin{array}{c}\text { Fine }\left(34 \mathrm{mg} / \mathrm{cm}^{2}\right) \\
(6 \mathrm{MV})\end{array}$ & $\begin{array}{c}\text { Regular }\left(70 \mathrm{mg} / \mathrm{cm}^{2}\right) \\
(6 \mathrm{MV})\end{array}$ & $\begin{array}{c}\text { Fast B }\left(133 \mathrm{mg} / \mathrm{cm}^{2}\right) \\
(6 \mathrm{MV})\end{array}$ & $\begin{array}{c}\text { Fast B }\left(133 \mathrm{mg} / \mathrm{cm}^{2}\right) \\
(15 \mathrm{MV})\end{array}$ \\
\hline Sensitivity (e/MU) & $13 \times 10^{6}$ & $60.9 \times 10^{6}$ & $128.2 \times 10^{6}$ & $107.6 \times 10^{6}$ \\
\hline \hline
\end{tabular}

for), which may be more pronounced for the thinner screens, and which results in the reduced differences observed between the total system MTFs for the various screens.

\section{Noise power spectra}

Figure 5 shows one-dimensional noise power spectra corresponding to the various combinations of screen type, beam energy, and irradiation (MU), obtained from both measurements and calculations [performed using Eq. (3)]. At $6 \mathrm{MV}$, there is good agreement between calculations and measure- ments for the Fast B screen, both at 1 and 2 MU. This agreement is observed both in magnitude (which is determined by the system gain) and the shape (which is determined by the optical screen MTF and the photodiode MTF, $T_{4}$ and $T_{6}$, respectively). In the determination of the calculated NPS [using Eq. (3)], contributions from the total additive noise were estimated empirically by means of the dark NPS and were included in the calculation. For all screens and energies, the corresponding dark NPS was found to be approximately constant $\left(\sim 2 \times 10^{9} \mathrm{~mm}^{2}\right)$ and independent of spatial frequency.
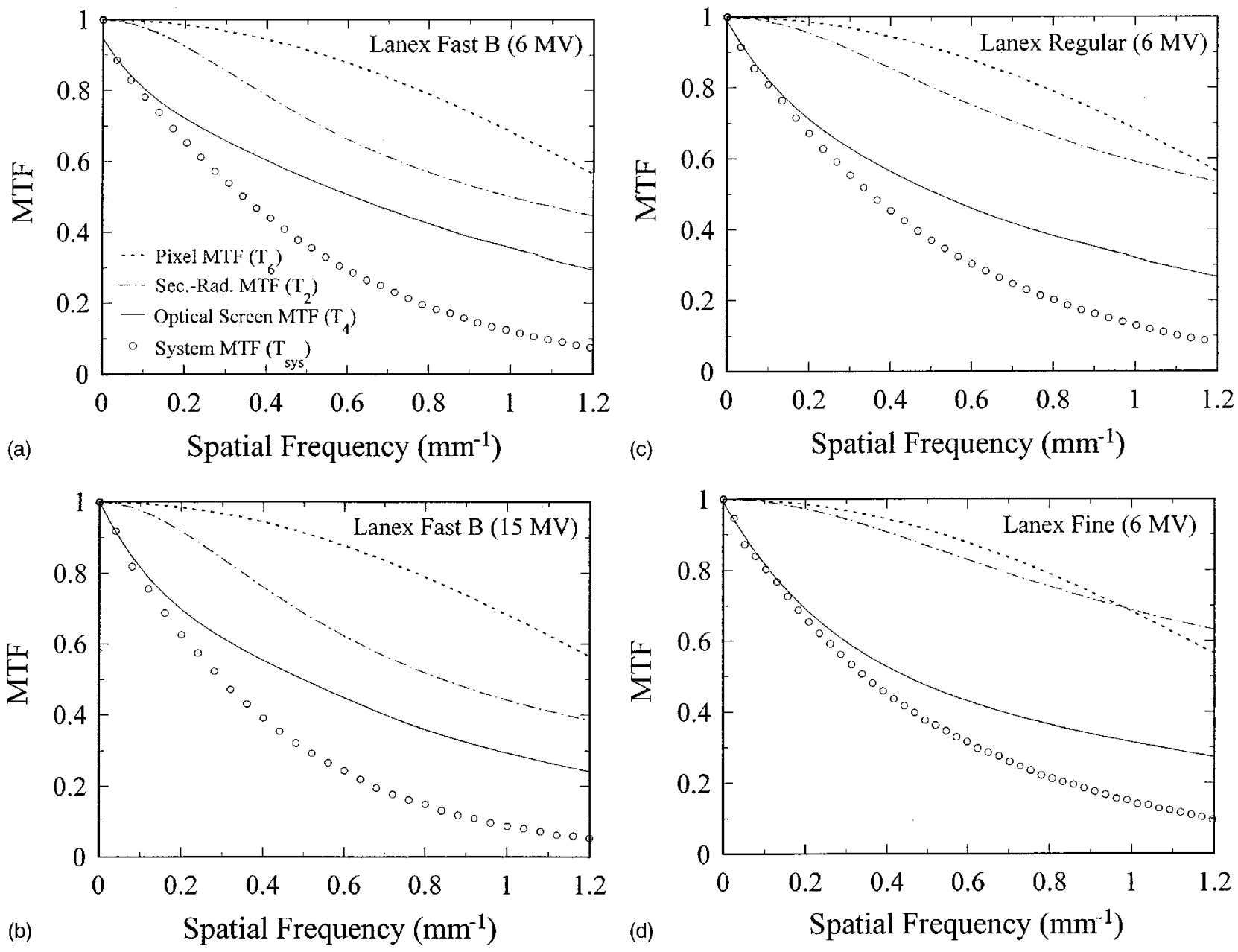

FIG. 4. Modulation transfer functions (MTF) associated with the prototype megavoltage imager determined for the following screens and energies: (a) Fast B at $6 \mathrm{MV}$; (b) Fast B at $15 \mathrm{MV}$; (c) Regular at $6 \mathrm{MV}$; and (d) Fine at $6 \mathrm{MV}$. In each plot, the measured total system MTF ( $T_{\text {sys }}$ ) is shown (open circles) along with its respective components: the secondary-radiation MTF, $T_{2}$, (dash-dotted line), the optical screen MTF, $T_{4}$ (solid line), and the photodiode aperture MTF, $T_{6}$, (dotted line). While $T_{2}$ and $T_{6}$ are calculated, $T_{4}$ is derived from the division of $T_{\text {sys }}$ by the product of $T_{2}$ and $T_{6}$. Note that the curves shown for $T_{4}$ represent fits to the derived data points. 


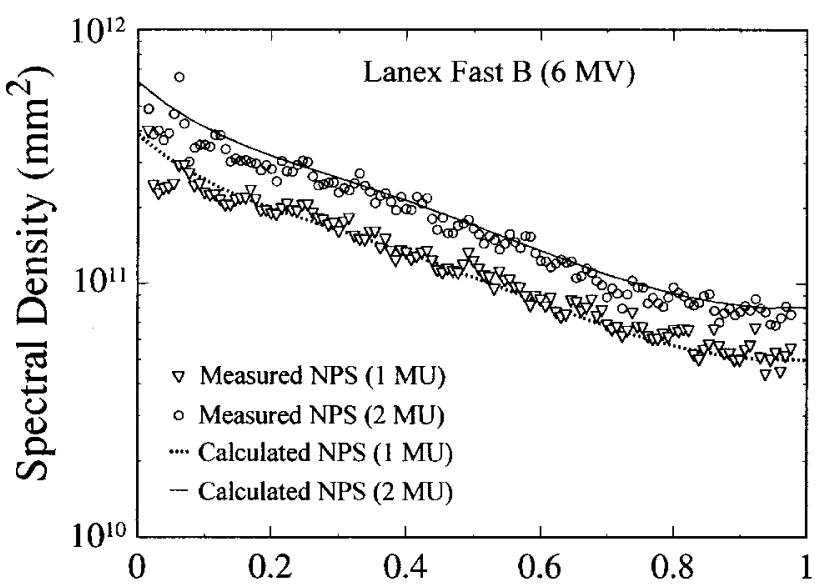

(a)

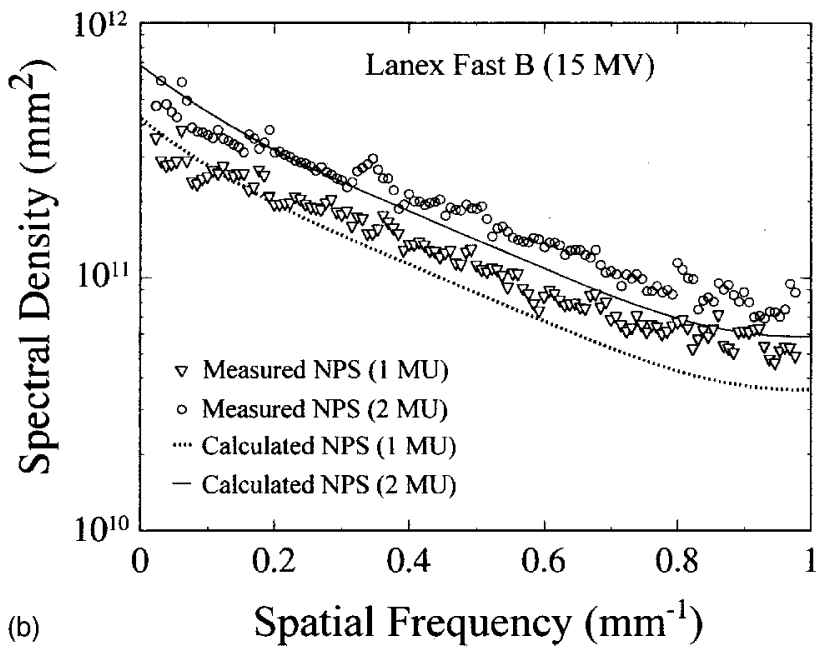

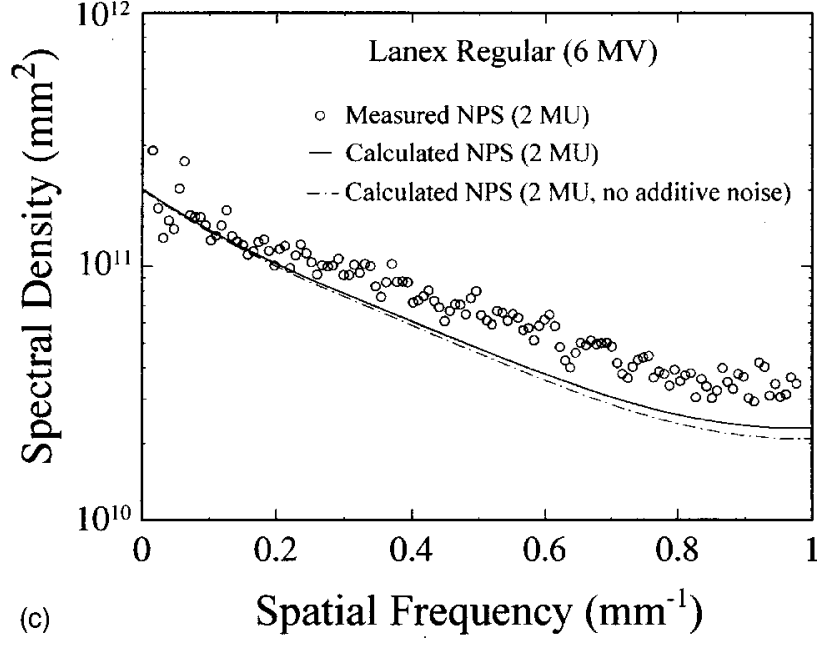

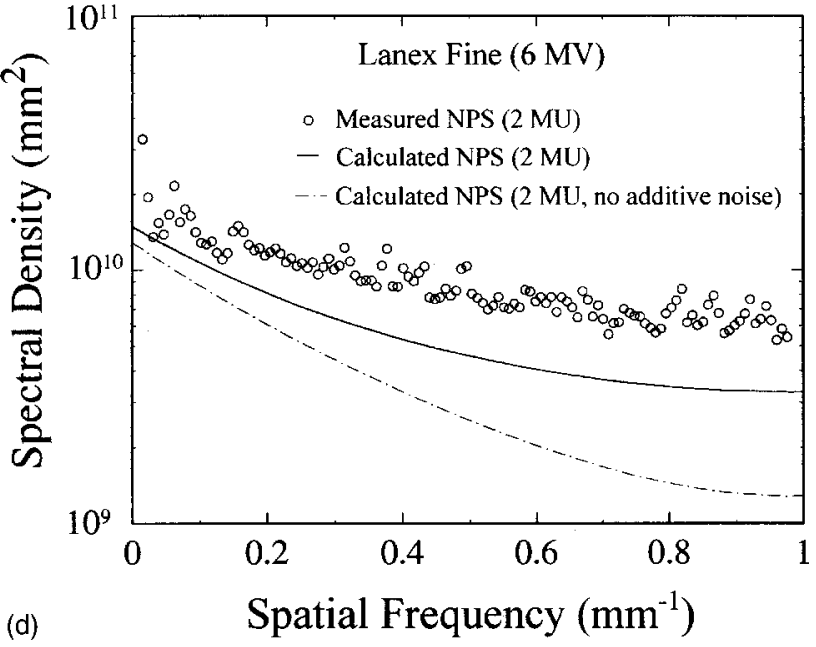

FIG. 5. Measured and theoretical one-dimensional NPS for the prototype imager determined for the following screens, energies, and irradiations: (a) Fast B at $6 \mathrm{MV}$ and 1,2 MU; (b) Fast B at $15 \mathrm{MV}$ and 1,2 MU; (c) Regular at $6 \mathrm{MV}$ and $2 \mathrm{MU}$; and (d) Fine at $6 \mathrm{MV}$ and $2 \mathrm{MU}$. Measurements of NPS at 1 and $2 \mathrm{MU}$ are indicated by open triangles and open circles, respectively. The calculated NPS at 1 and 2 MU are indicated by dashed and solid lines, respectively. For (c) and (d), NPS calculated before the addition of total additive noise (dark NPS) is indicated by dash-dotted lines.

In the case of the Fast B screen, the contribution of the dark NPS to the total NPS was negligible both at 6 and 15 MV due to the relatively high signal output of the screen.

In Fig. 5(b), NPS data obtained at 15 MV for the Fast B screen is shown for 1 and $2 \mathrm{MU}$. In this case, a comparison between measured and calculated NPS reveals a fair agreement in magnitude but a small difference in shape both at 1 and 2 MU. This difference is most probably due to an underestimation of the screen optical MTF, $T_{4}$. For both Figs. 5(a) and 5(b), a comparison of the $1 \mathrm{MU}$ and $2 \mathrm{MU}$ data reveals that NPS does not scale by a factor of 2 , as might be expected given the increase in dose. The reduced difference is due to increased charge trapping for the higher signal data (2 $\mathrm{MU}){ }^{23}$

For the Regular screen data at $2 \mathrm{MU}$, shown in Fig. 5(c), fairly good agreement between measured and calculated NPS is observed at low frequencies. At higher frequencies, the calculation underestimates the measurement, probably due to an underestimation of the derived optical screen MTF, $T_{4}$. For the Fine screen data, shown in Fig. 5(d), a more substan- tial difference between calculated and measured NPS is observed. Unlike for the Fast B and Regular screens, the difference at zero frequency is unexpected, since the overall effect of system gain in the calculations was determined by fitting the measured sensitivity. The observed underestimation could be due to an underestimation of the Poisson excess, $\epsilon_{g_{3}}$. In this case, $\epsilon_{g_{3}}$, which is derived from the Swank factor, accounts only for variations in the absorbed energy distributions in the phosphor. Any variations caused by optical light transport are neglected. At diagnostic energies, it was found that the Fine screen exhibits much larger noise contributions from optical light transport than the other two phosphor screens, ${ }^{39}$ hence the probable reason for the observed underestimation of the Poisson excess. At higher frequencies, underestimation of the optical screen MTF, $T_{4}$, may also contribute to the observed discrepancy between measured and calculated NPS.

In both Figs. 5(c) and 5(d), the effect of total additive noise in the calculations is illustrated by showing NPS re- 

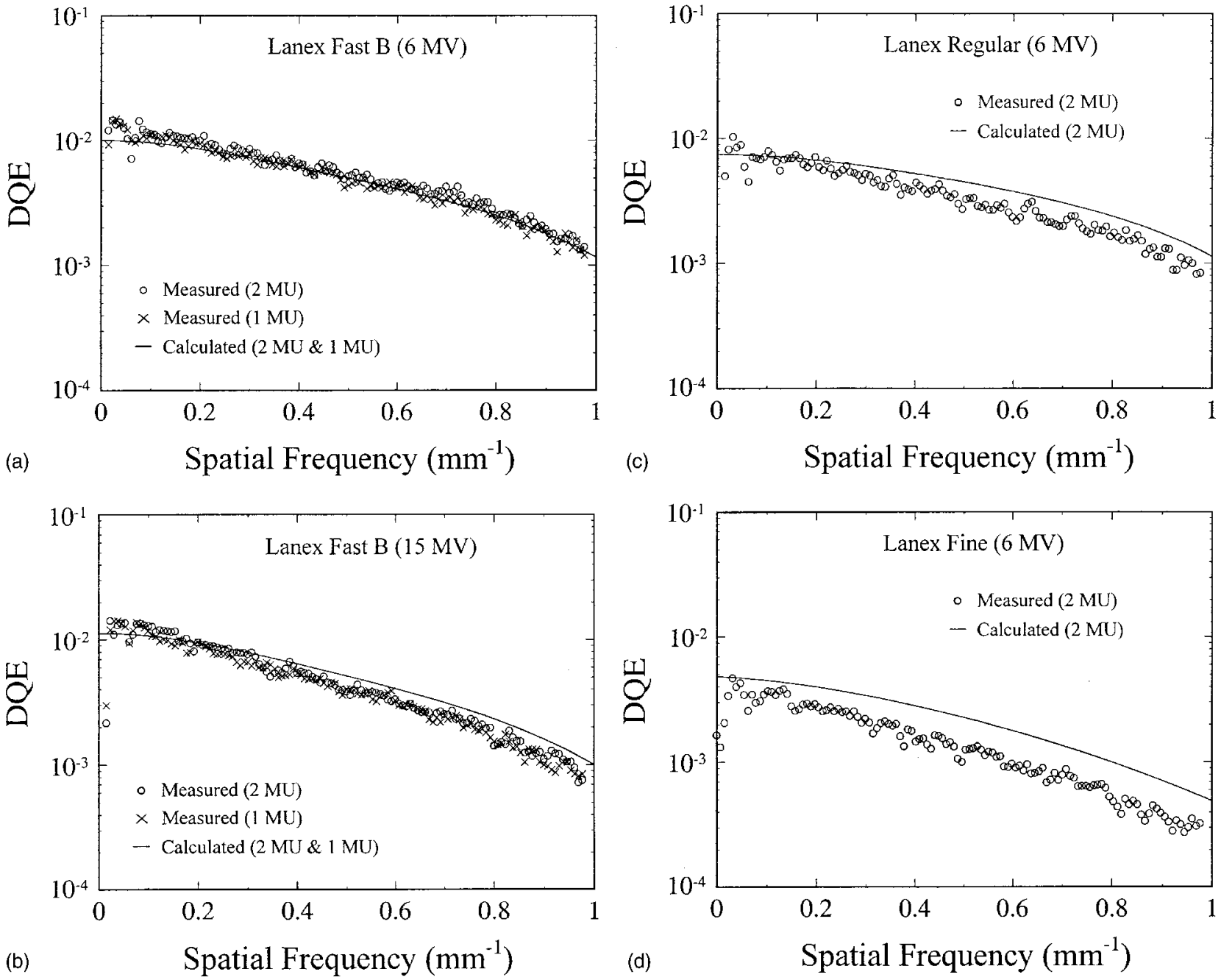

FIG. 6. Measured and theoretical DQE for the prototype imager determined for the following screens, energies, and irradiations: (a) Fast B at 6 MV and 1, 2 MU; (b) Fast B at 15 MV and 1, 2 MU; (c) Regular at 6 MV and 2 MU; and (d) Fine at 6 MV and 2 MU. The measured DQE results for 1 and 2 MU are indicated by crosses and open circles, respectively. Calculated DQE results are indicated by solid lines. For the Fast B screen [(a) and (b)], calculated DQE results for both 1 and $2 \mathrm{MU}$ are indicated by a single line since their corresponding values are identical.

sults before and after the addition of this component. This effect is relatively more pronounced in the case of the Fine screen due to its lower signal output. A comparison of the NPS data for the various screens at $6 \mathrm{MV}$ (2 MU) shows increasing magnitude with increasing phosphor thickness, mainly due to the increase in the light output, as demonstrated by the sensitivity results.

\section{Detective quantum efficiencies}

Figure 6 shows DQE results from measurements as well as from the corresponding cascaded systems calculations for the prototype megavoltage imager employing the various phosphor screens. For all conditions shown, the zerofrequency DQE was found to be dominated by the quantum detection efficiency, $\overline{g_{1}}$, and Swank factor of the converter, indicating that system performance is mainly limited by the performance of the x-ray converter (at zero frequency). In Fig. 6(a), measured DQE for the Fast B screen at $6 \mathrm{MV}$ is shown to be in good agreement with the calculations both at
1 and $2 \mathrm{MU}$. While the incident radiation signal is almost doubled from 1 to $2 \mathrm{MU}$, the DQE remains unchanged-a clear indication that the imager in this configuration is inputquantum-limited (i.e., the performance is only limited by the $\mathrm{x}$-ray quantum noise and not by other noise factors). A similar observation is made in the case of the $15 \mathrm{MV}$ data shown in Fig. 6(b). In this case, however, a discrepancy is observed between measured and calculated DQE, probably due to an underestimation of the optical screen MTF of the converter, $T_{4}$ (as seen in the NPS results). DQE results for the Regular screen, shown in Fig. 6(c), exhibit agreement between the calculation and measurement at low spatial frequencies. At high frequencies, however, the calculated DQE overestimates the measurements - a difference probably caused by an underestimation of the optical screen MTF, $T_{4}$. In the case of the Fine screen, DQE results shown in Fig. 6(d) exhibit an overestimation of the measurement at all spatial frequencies. As explained in the previous section, this difference is most likely caused by the underestimation of both the Poisson excess $\left(\epsilon_{g_{3}}\right)$ and the optical screen MTF, $T_{4}$. A 
comparison of the DQE performance of the prototype imager for the various screens at $6 \mathrm{MV}$ [Figs. 6(a), 6(c), and 6(d)] reveals a decrease in DQE for decreasing screen thickness. This is primarily due to the decrease in quantum detection efficiency, $\overline{g_{1}}$, with thinner screens.

\section{SUMMARY AND CONCLUSIONS}

After about 14 years of aggressive development by our group and others, active matrix flat-panel imagers have recently become commercially available for radiotherapy imaging. In this paper, a comprehensive evaluation of the signal and noise performance of a large-area prototype AMFPI specifically developed for this application is reported. The study involved both empirical and theoretical investigations of the signal, NPS and DQE properties of the imager under a variety of imaging conditions. The imager comprises a pixelated array, incorporating photodiodes and thin-film transistors, an $\mathrm{X}$-ray converter, and an associated electronic acquisition system. In this study, the performance of the imager with three different converters (Lanex Fast B, Lanex Regular, and Lanex Fine) was obtained. The empirical system performance involved measurements of sensitivity, MTF and NPS from which the DQE was derived. Theoretical calculations were performed and compared to measured results. The calculations employed a cascaded systems analysis in which the imaging system was divided into a series of stages. In addition, some of the parameters employed in the calculations (such as the quantum detection efficiency, Swank factor, and the spatial spreading induced by the secondary radiation within the converter) were obtained through EGS4 Monte Carlo simulations.

For a given beam energy and for the various imager configurations, measured sensitivity was found to increase with increasing thickness of the phosphor screen due to the increase in quantum detection efficiency and quantum gain of the screen. For a given screen, the lower-energy beam yielded a higher sensitivity. Comparisons of these sensitivity measurements with the corresponding calculations were used to determine the conversion efficiencies of the various phosphor screens.

Measurements of system MTF for the various imager configurations indicate that as phosphor thickness increases, the spatial resolution of the system degrades only slightlyunlike the strong reduction of MTF observed at diagnostic energies. This reduced dependence is believed to be partially due to the blurring induced by the greater range of secondary electrons generated in the converter at megavoltage energies. The measured MTF at $15 \mathrm{MV}$ is lower than that at $6 \mathrm{MV}$, as expected, due to the increased range of secondary electrons at this higher beam energy.

Measurements of one-dimensional NPS indicate that this quantity increases with the amount of input radiation and with the thickness of the phosphor screen, due to differences in phosphor light output. The corresponding DQE values for the various converters were obtained using the measured MTF and NPS and the calculated x-ray beam fluence. In the case of the Fast B phosphor screen, the DQE results at 1 and
$2 \mathrm{MU}$ were very similar at 6 and at $15 \mathrm{MV}$. The fact that DQE is independent of the magnitude of the irradiation implies that, with this screen, the prototype imager is inputquantum-limited at 1 and $2 \mathrm{MU}$, even at an extended SDD of $200 \mathrm{~cm}$. The maximum DQE obtained is around 1\%-a limit imposed by the low x-ray detection efficiency of the phosphor (Fast B)+copper. For the other phosphor screens (Regular and Fine), the corresponding DQE at $2 \mathrm{MU}$ is lower than that for the Fast B due to their lower detection efficiencies.

The DQE performance was compared to calculations based on a cascaded systems model in which the imaging system is represented by a series of gain and spatial spreading stages. The statistical characteristics of each stage were numerically quantified and incorporated into a mathematical expression for the DQE. In this model, compared to similar analyses performed at diagnostic energies on similar indirect detection flat-panel imagers, an additional spreading stage characterized by a MTF was added to account for the scattering of the secondary radiation within the converter. In this case, the MTF of the system was conceptually divided into three distinct MTF components: a component associated with the lateral spread of energy deposited by the secondary electrons generated by the interacting incident radiation; a component related to the scatter of individual light photons within the phosphor; and a component defined by the photodiode aperture. Lacking an analytical model for the blur caused by secondary electrons, the corresponding MTF was determined using Monte Carlo techniques based on EGS4 simulations. The optical MTF of the screen was deduced by dividing the measured system MTF by the product of the MTF of the secondary electrons and the MTF of the photodiode aperture. For the AMFPI configurations and imaging conditions used, calculations of DQE were compared to the corresponding empirical results. From these comparisons, it was found that the introduction of the spreading stage that accounted for blur caused by scatter of secondary electrons within the converter contributed toward improved agreement between model predictions and all empirical results. The agreement was reasonably good at $6 \mathrm{MV}$ for the thickest phosphor screen (Lanex Fast B). However, agreement was poorer for thinner screens. This could be due to the failure of the MTF model to account for some additional blurring mechanism that is less significant for the thickest screen. Finally, for the thinnest screen (Lanex Fine), differences in magnitude between calculated and measured DQE at zero frequency are most likely due to an overestimation of the Swank factor in the Monte Carlo simulation, which accounts for effects related to x-ray energy distributions, but ignores effects associated with optical transport in the phosphor.

Overall, the performance of present indirect detection AMFPIs employing a thick, commercially available phosphor screen (Lanex Fast B) is shown to exhibit a maximum DQE at megavoltage energies of approximately $1 \%$ - a value mainly limited by the inefficient use of incident radiation by the converter. (This screen configuration is employed in current commercial AMFPI megavoltage systems. ${ }^{4}$ ) Their performance is, however, superior to that of conventional radio- 
therapy film systems, as previously demonstrated in an earlier observer-dependent study. ${ }^{6}$ In this earlier study, even with a thinner phosphor screen (Lanex Regular), the AMFPI provided higher quality, clinically useful images at a reduced dose- thus contributing to the evidence that this technology may replace film as the gold standard for megavoltage image quality. In comparison to other established electronic portal imagers such as mirror+lens-coupled camera-based systems, the measured DQEs of indirect detection AMFPIs are superior due to their higher light coupling efficiencies, with coupling efficiencies of the order of $\sim 50 \%$ for AMFPIs compared to $\sim 0.1 \%$ to $0.01 \%$ for camera-based systems. ${ }^{4,20}$

In terms of DQE performance, the use of the thickest phosphor screen resulted in the best performance, as expected. However, assessing the performance of thinner phosphor screens allowed comparisons of the cascaded systems model with empirical data under a wider variety of conditions. Despite the good agreement obtained between measurements and model predictions for the imager configuration with the thick screen, the failure of the model to accurately predict other configurations with thinner screens indicates the need for improvements to the model. One improvement would involve making a more accurate estimate of the screen Swank factor. Another improvement would be to reconfigure stages 2 and 3 so as to more accurately describe the simultaneous process of energy deposition and light production associated with secondary radiation within the converter. For example, the recently reported Monte Carlo-based methodology of Lachaine et al., ${ }^{42}$ which has been developed to examine the DQE of thick phosphors, could be of interest in the present context. In conclusion, empirical examinations of active matrix flat-panel imagers for radiotherapy, such as that presented in this paper, provide objective, quantitative measures of how well the technology is performing. Furthermore, the availability of such information facilitates the development and validation of sophisticated, detailed models, such as the cascaded-systems-based model in this paper. Such models are currently being used by our group to explore strategies of significantly enhancing the performance of AMFPI technology in radiotherapy imaging beyond the relatively modest DQE levels attained by existing devices.

\section{ACKNOWLEDGMENTS}

The authors extend their sincere gratitude to Codrin Cionca for assistance with the measurements and to Ian Cunningham for valuable discussions concerning the model. This work was supported by Grant No. R01-CA51397 from the National Institutes of Health.

\footnotetext{
a) Author to whom correspondence should be addressed. Telephone: 734936-4309; fax: 734-936-7859; electronic mail: elmohri@umich.edu ${ }^{1}$ R. A. Street, S. Nelson, L. E. Antonuk, and V. Perez Mendez, "Amorphous silicon sensor arrays for radiation imaging," Mater. Res. Soc. Symp. Proc. 192, 441-452 (1990).

${ }^{2}$ W. Zhao, I. Blevis, S. Germann, and J. A. Rowlands, "Digital radiology using active matrix readout of amorphous selenium: construction and evaluation of a prototype real-time detector," Med. Phys. 27, 1834-1843 (1997).
}

${ }^{3}$ L. E. Antonuk, J. Boudry, W. Huang, D. L. McShan, E. J. Morton, J. Yorkston, M. J. Longo, and R. A. Street, "Demonstration of megavoltage and diagnostic x-ray imaging with hydrogenated amorphous silicon arrays," Med. Phys. 19, 1455-1466 (1992).

${ }^{4}$ L. E. Antonuk, "Electronic portal imaging devices (EPIDs): A review and historical perspective of contemporary technologies and research," accepted for publication in Phys. Med. Biol. J.

${ }^{5}$ L. E. Antonuk, J. Yorkston, W. Huang, H. Sandler, J. H. Siewerdsen, and Y. El-Mohri, "Megavoltage imaging with a large area, flat-panel, amorphous silicon imager," Int. J. Radiat. Oncol., Biol., Phys. 36, 661-672 (1996).

${ }^{6}$ L. E. Antonuk, Y. El-Mohri, W. Huang, K.-W. Jee, J. H. Siewerdsen, M. Maolinbay, V. E. Scarpine, H. Sandler, and J. Yorkston, "Initial performance evaluation of an indirect-detection, active matrix flat-panel imager (AMFPI) prototype for megavoltage imaging," Int. J. Radiat. Oncol., Biol., Phys. 42, 437-454 (1998).

${ }^{7}$ L. E. Antonuk, J. Boudry, W. Huang, K. L. Lam, E. J. Morton, R. K. Ten Haken, J. Yorkston, and N. H. Clinthorne, "Thin-film, flat-panel, composite imagers for projection and tomographic imaging," IEEE Trans. Med. Imaging 13, 482-490 (1994).

${ }^{8}$ L. E. Antonuk, J. Yorkston, J. Boudry, M. J. Longo, J. Jimenez, and R. A. Street, "Development of hydrogenated amorphous silicon sensors for high energy photon radiotherapy imaging," IEEE Trans. Nucl. Sci. 37, 165-170 (1990).

${ }^{9}$ L. E. Antonuk, J. Boudry, J. Yorkston, C. F. Wild, M. J. Longo, and R. A. Street, "Radiation damage studies of amorphous silicon photodiode sensors for applications in radiotherapy x-ray imaging," Nucl. Instrum. Methods Phys. Res. A 299, 143-146 (1990).

${ }^{10}$ L. E. Antonuk, J. Yorkston, C. W. Kim, W. Huang, E. J. Morton, M. J. Longo, and R. A. Street, "Light response characteristics of amorphous silicon arrays for megavoltage and diagnostic imaging," Mater. Res. Soc. Symp. Proc. 219, 531-536 (1991).

${ }^{11}$ L. E. Antonuk, J. Yorkston, W. Huang, J. Boudry, E. J. Morton, M. J. Longo, and R. A. Street, "Radiation response characteristics of amorphous silicon arrays for megavoltage radiotherapy imaging," IEEE Trans. Nucl. Sci. 39, 1069-1073 (1992).

${ }^{12}$ L. E. Antonuk, J. Yorkston, W. Huang, J. Boudry, E. J. Morton, M. Longo, and R. A. Street, "Factors affecting image quality for megavoltage and diagnostic X-ray $a$-Si:H imaging arrays," Mater. Res. Soc. Symp. Proc. 258, 1069-1074 (1992).

${ }^{13}$ J. Yorkston, L. E. Antonuk, W. Huang, and R. A. Street, "Photoresponse linearity of $a$-Si:H imaging pixels,” Mater. Res. Soc. Symp. Proc. 297, 951-956 (1993).

${ }^{14}$ J. Boudry and L. E. Antonuk, "Current-noise-power-spectra for amorphous silicon photodiode sensors," Mater. Res. Soc. Symp. Proc. 297, 975-980 (1993).

${ }^{15}$ J. M. Boudry and L. E. Antonuk, "Current-noise-power-spectra of amorphous silicon thin-film transistors," J. Appl. Phys. 76, 2529-2534 (1994).

${ }^{16} \mathrm{~J}$. M. Boudry and L. E. Antonuk, "Radiation damage of amorphous silicon, thin-film, field-effect transistors," Med. Phys. 23, 743-754 (1996).

${ }^{17}$ Y. El-Mohri, L. E. Antonuk, J. Yorkston, K.-W. Jee, M. Maolinbay, K. L. Lam, and J. H. Siewerdsen, "Relative dosimetry using active matrix flatpanel imager (AMFPI) technology," Med. Phys. 26, 1530-1541 (1999).

${ }^{18}$ J. R. D. Earnhart and E. L. Chaney, "Modulation transfer function for a large-area amorphous silicon image receptor," Phys. Med. Biol. 42, 2531-2540 (1997).

${ }^{19}$ D. G. Drake, D. A. Jaffray, and J. Wong, "A prototype amorphous silicon array based radiotherapy portal imager," Proc. SPIE 3032, 32-41 (1997).

${ }^{20} \mathrm{P}$. Munro and D. C. Bouius, "X-ray quantum limited portal imaging using amorphous silicon flat-panel arrays," Med. Phys. 25, 689-702 (1998).

${ }^{21}$ I. A. Cunningham, M. S. Westmore, and A. Fenster, "A spatial-frequency dependent quantum accounting diagram and detective quantum efficiency model of signal and noise propagation in cascaded imaging systems," Med. Phys. 21, 417-427 (1994).

${ }^{22}$ E. J. Morton, L. E. Antonuk, J. E. Berry, W. Huang, P. Mody, and J. Yorkston, "A data acquisition system for flat-panel imaging arrays," IEEE Trans. Nucl. Sci. 41, 1150-1154 (1994).

${ }^{23}$ L. E. Antonuk, Y. El-Mohri, J. H. Siewerdsen, J. Yorkston, W. Huang, V. E. Scarpine, and R. A. Street, "Empirical investigation of the signal performance of a high-resolution, indirect detection, active matrix flatpanel imager (AMFPI) for fluoroscopic and radiographic operation," Med. Phys. 24, 51-70 (1997). 
${ }^{24}$ H. Fujita, K. Doi, and M. L. Giger, "Investigation of basic imaging properties in digital radiography. 6. MTFs of II-TV digital imaging systems," Med. Phys. 12, 713-720 (1985).

${ }^{25}$ J. C. Dainty and R. Shaw, Image Science: Principles, Analysis and Evaluation of Photographic Type Imaging Processes (Academic, London, 1974).

${ }^{26}$ M. L. Giger, K. Doi, and C. E. Metz, "Investigation of basic imaging properties in digital radiography. 2. Noise Wiener spectrum," Med. Phys. 11, 797-805 (1984).

${ }^{27}$ A. D. A. Maidment and M. Yaffe, "Analysis of the spatial frequencydependent DQE of optically coupled digital mammography detectors," Med. Phys. 21, 721-729 (1994).

${ }^{28}$ J. H. Siewerdsen, L. E. Antonuk, Y. El-Mohri, J. Yorkston, W. Huang, J. M. Boudry, and I. A. Cunningham, "Empirical and theoretical investigation of the noise performance of indirect detection, active matrix flatpanel imagers (AMFPIs) for diagnostic radiology,” Med. Phys. 24, 71-89 (1997).

${ }^{29}$ J. H. Siewerdsen, L. E. Antonuk, Y. El-Mohri, J. Yorkston, W. Huang, and I. A. Cunningham, "Signal, noise power spectrum, and detective quantum efficiency of indirect-detection flat-panel imagers for diagnostic radiology," Med. Phys. 25, 614-628 (1998).

${ }^{30}$ J. P. Bissonnette, I. A. Cunningham, D. A. Jaffray, A. Fenster, and P. Munro, "A quantum accounting and detective quantum efficiency analysis for video-based portal imaging," Med. Phys. 24, 815-826 (1997).

${ }^{31}$ D. G. Drake, D. A. Jaffray, and J. W. Wong, "Characterization of a fluoroscopic imaging system for kV and MV radiotherapy," Med. Phys. 27, 898-905 (2000).

${ }^{32}$ M. Rabbani, R. Shaw, and R. Van Metter, "Detective quantum efficiency of imaging systems with amplifying and scattering mechanisms," J. Opt. Soc. Am. A 4, 895-901 (1987).
${ }^{33}$ J. Yao and I. Cunningham, "Parallel-cascades: New ways to describe noise transfer in medical imaging systems," Med. Phys. 28, 2020-2038 (2001).

${ }^{34}$ I. Cunningham, "Applied linear-system theory," in Handbook of Medical Imaging, Volume 1. Physics and Psychophysics, edited by J. Beutel, H. L. Kundel, and R. L. Van Metter (SPIE, Bellingham, WA, 2000).

${ }^{35}$ D. W. O. Rogers, "Fluence to dose equivalent conversion factors calculated with EGS3 for electrons from $100 \mathrm{keV}$ to $20 \mathrm{GeV}$ and photons from $11 \mathrm{keV}$ to $20 \mathrm{GeV}$," Health Phys. 46, 891-914 (1984).

${ }^{36}$ D. Sheikh-Bagheri, "Monte Carlo study of photon beams from medical linear accelerators: Optimization, benchmarks and spectra," Thesis, Department of Physics, Carleton University, 1998.

${ }^{37}$ D. Jaffray, J. J. Batista, A. Fenster, and P. Munro, "Monte Carlo studies of $\mathrm{x}$-ray energy absorption and quantum noise in megavoltage transmission radiography," Med. Phys. 22, 1077-1088 (1995).

${ }^{38}$ G. Zweig and D. A. Zweig, "Radioluminescent imaging: Factors affecting total light output," Proc. SPIE 419, 297-301 (1979).

${ }^{39}$ D. P. Trauernicht and R. Van Metter, "The measurement of conversion noise in x-ray intensifying screens," Proc. SPIE 914, 100-116 (1988).

${ }^{40}$ C.-M. Ma, P. Reckwerdt, M. Holmes, D. W. O. Rogers, B. Geiser, and B. Walters, "DOSXYZ users manual," NRCC Report PIRS-509B, 1995.

${ }^{41}$ L. E. Antonuk, Y. El-Mohri, A. Hall, K.-W. Jee, M. Maolinbay, S. C. Nassif, X. Rong, J. H. Siewerdsen, Q. Zhao, and R. Weisfield, "A largearea, $97 \mu \mathrm{m}$ pitch, indirect-detection, active matrix, flat-panel imager (AMFPI)," Proc. SPIE 3336, 2-13 (1998).

${ }^{42}$ M. Lachaine and B. G. Fallone, "Cascade analysis for medical imaging detectors with stages involving both amplification and dislocation processes," Med. Phys. 28, 501-507 (2001). 